This item was submitted to Loughborough's Research Repository by the author.

Items in Figshare are protected by copyright, with all rights reserved, unless otherwise indicated.

\title{
Invariant manifold based output-feedback sliding mode control for systems with mismatched disturbances
}

PLEASE CITE THE PUBLISHED VERSION

https://doi.org/10.1109/tcsii.2020.3011458

\section{PUBLISHER}

Institute of Electrical and Electronics Engineers (IEEE)

\section{VERSION}

AM (Accepted Manuscript)

\section{PUBLISHER STATEMENT}

(c) 2020 IEEE. Personal use of this material is permitted. Permission from IEEE must be obtained for all other uses, in any current or future media, including reprinting/republishing this material for advertising or promotional purposes, creating new collective works, for resale or redistribution to servers or lists, or reuse of any copyrighted component of this work in other works.

\section{LICENCE}

All Rights Reserved

\section{REPOSITORY RECORD}

Zhang, Lu, Jun Yang, Shihua Li, and Xinghuo Yu. 2020. "Invariant Manifold Based Output-feedback Sliding Mode Control for Systems with Mismatched Disturbances". Loughborough University. https://hdl.handle.net/2134/15074622.v1. 


\title{
Invariant Manifold Based Output-Feedback Sliding Mode Control for Systems with Mismatched Disturbances
}

\author{
Lu Zhang, Jun Yang, Senior Member, IEEE, Shihua Li, Fellow, IEEE, and Xinghuo Yu, Fellow, IEEE
}

\begin{abstract}
This paper proposes an invariant manifold based output-feedback sliding mode control (SMC) strategy for systems with mismatched disturbances to achieve asymptotic tracking and disturbance rejection. Different from the existing outputfeedback SMC methods, the invariant manifold is employed to transform the multiple mismatched disturbances into matched ones, which can provide full system dynamics for controller design to improve accuracy. An observer is developed to estimate unmeasurable states, then an output-feedback sliding mode controller is proposed. Moreover, the switching gain of the controller adaptively changes with the estimation error, which reduces chattering in SMC. Experiments on a converter-driven DC motor system verify the superiority of the proposed method.
\end{abstract}

Index Terms-Sliding mode control, output-feedback, mismatched disturbances, invariant manifold.

\section{INTRODUCTION}

Sliding mode control has been paid much attention [1] [2] due to its robustness to matched uncertainties. [3] and [4] propose sliding-mode neuro-controller and fractional-order SMC for systems with matched disturbances, respectively. However, mismatched disturbances widely exist in practical systems, such as spacecraft system [5], DC-DC converters [6] and so on. Various control approaches are developed to handle mismatched disturbances, such as integral SMC [7], disturbance observer-based approach [8], anti-disturbance bumpless transfer control [9]. The results are mostly implemented by full-state measurement, which would be difficult in practice due to cost and installation limitations. Therefore, it is of great importance to investigate output-feedback SMC to reject mismatched disturbances.

The existing output-feedback SMC methods are mainly divided into two categories. The first strategy is depending on the outputs directly or on an output-based dynamic compensator indirectly [10]. In [11], a rate bumpless transfer controller for switched linear system with output feedback is proposed using multiple Lyapunov functions and linear matrix inequality (LMI). [5] investigates output-feedback SMC for spacecraft hovering system, and the robust stability in the sliding phase

The work was supported in part by the National Natural Science Foundation of China under Grant 61973080 and Grant 61973081. (Corresponding author: Jun Yang.)

L. Zhang, J. Yang, and S. Li are with the School of Automation, Southeast University, Key Laboratory of Measurement and Control of CSE Ministry of Education, Nanjing 210096, China (e-mail: zhanglu@seu.edu.cn; j.yang84@seu.edu.cn; 1sh@seu.edu.cn).

$\mathrm{X}$. Yu is with the School of Electrical and Computer Engineering, RMIT University, Melbourne, VIC 3001, Australia (e-mail: x.yu@rmit.edu.au). is derived from LMI. These approaches generally require that the controller gains are large enough to suppress disturbances, which sacrifices the nominal performance of the system. The second strategy is observer-based SMC [12], the key idea of which is taking $n$ th-order differentiations of the output for a system with relative degree $n$ and lumping all the rest dynamics except for the integral chain as lumped disturbances. Then, an observer is developed to reconstruct unmeasurable states. [13] proposes a higher-order sliding mode observer to achieve output-feedback, but the controller is complicated especially for higher-order systems, which brings difficulties in implementation. Recently, extended state observer (ESO) [14] based output-feedback SMC is paid attention due to its simpleness in implementation [15] [16]. The existing observerbased method is relatively conservative. Since there is state information in the lumped disturbance, the observer poles should be relatively large to obtain satisfactory performance, which will lead to a drastic rise of observer gains and significant noise amplification especially for higher-order systems. As a result, the frequently switching in control signal will be serious, which has negative effects on the actuator [17].

By utilizing steady state of control input, [18] proposes a robust granular feedback linearization method to achieve asymptotic tracking and disturbance rejection, which requires full-state measurement or tracking differentiator in implementation. Inspired by [18] and the idea of invariant manifold in output regulation [19], this paper aims to address the aforementioned problems in output-feedback SMC as well as ensuring satisfactory control performance and disturbance rejection ability for systems subject to mismatched disturbances. The main contributions of this paper are summarized as follows: 1) the transformation based on the invariant manifold reduces the burden on the observer, which admits lower observer poles to achieve satisfactory performance, thus largely attenuates measurement noises; 2) the chattering problem in SMC can be attenuated to a large extent since the switching gain changes with the estimation error adaptively; 3 ) the proposed approach can effectively compensate the influence caused by unknown time-derivatives of the reference signal without any tracking differentiator, which leads to a more concise control structure and simple implementation.

\section{PRELIMINARIES}

Firstly, we recall the following lemma that will play an important role in the subsequent analysis. 
Lemma 1. [20] Assume $A \in \mathbb{R}^{n \times n}$ is Hurwitz, then there exists a positive scalar $c>0$ such that $\left\|e^{A t}\right\| \leq c e^{\frac{\lambda_{\max }(A)}{2} t}$, where $\lambda_{\max }(A)=\max _{i}\left\{\operatorname{Re}\left(\lambda_{i}(A)\right)\right\}$.

\section{A. Problem Formulation}

Consider a system with mismatched disturbances:

$$
\begin{aligned}
\dot{\boldsymbol{x}} & =\boldsymbol{A} \boldsymbol{x}+\boldsymbol{B} u+\Omega, \\
y & =\boldsymbol{C} \boldsymbol{x},
\end{aligned}
$$

where $\boldsymbol{A}=\left[\begin{array}{cccc}a_{11} & a_{12} & \cdots & a_{1 n} \\ \vdots & \vdots & \ddots & \vdots \\ a_{n 1} & a_{n 2} & \cdots & a_{n n}\end{array}\right], \quad \boldsymbol{B}=\left[\begin{array}{c}0 \\ \vdots \\ 1\end{array}\right], \quad \boldsymbol{C}=$ $[1,0, \cdots, 0]_{1 \times n}$, with $\boldsymbol{x}=\left[x_{1}, x_{2}, \cdots, x_{n}\right]^{T} \in \mathbb{R}^{n}, u \in$ $\mathbb{R}$ and $y \in \mathbb{R}$ are system states, control input and system output, respectively. $\Omega$ is the unknown exosystem with $\Omega=\left[\omega_{1}, \omega_{2}, \cdots, \omega_{n}\right]^{T}, \omega_{i}(i=1,2, \cdots, n)$ are unknown disturbances. The control objective is to design an output feedback sliding mode controller which drives system output $y(t)$ to asymptotically track reference signal $r(t)$.

\section{B. Traditional Output-Feedback SMC}

Suppose the relative degree of system (1) is $m$ with $m \leq n$. Taking $m$ th-order derivatives of system output, one has

$$
\begin{aligned}
y & =\boldsymbol{C} \boldsymbol{x}, \dot{y}=\boldsymbol{C} \dot{\boldsymbol{x}}=\boldsymbol{C} \boldsymbol{A} \boldsymbol{x}+\boldsymbol{C} \Omega, \cdots, \\
y^{(m)} & =\boldsymbol{C} \boldsymbol{A}^{m} \boldsymbol{x}+\boldsymbol{C} \boldsymbol{A}^{m-1} \Omega+\cdots+\boldsymbol{C} \Omega^{(m-1)}+\boldsymbol{C} \boldsymbol{A}^{m-1}
\end{aligned}
$$

Denote $\varpi=\boldsymbol{C} \boldsymbol{A}^{m} \boldsymbol{x}+\boldsymbol{C} \boldsymbol{A}^{m-1} \Omega+\cdots+\boldsymbol{C} \Omega^{(m-1)}$ as the lumped disturbance and $\kappa=\boldsymbol{C} \boldsymbol{A}^{m-1} \boldsymbol{B}$. Letting $\boldsymbol{\nu}=$ $\left[\nu_{1}, \nu_{2}, \cdots, \nu_{m}, \nu_{m+1}\right]^{T}=\left[y, \dot{y}, \cdots, y^{(m-1)}, \varpi\right]^{T}$, we have [16] [21]

$$
\dot{\boldsymbol{\nu}}=\boldsymbol{A}_{1} \boldsymbol{\nu}+\boldsymbol{B}_{1} u+\Omega_{1}, \nu_{o}=\boldsymbol{C}_{1} \boldsymbol{\nu},
$$

where $\boldsymbol{A}_{1}=\left[\begin{array}{ccccc}0 & 1 & 0 & \cdots & 0 \\ \vdots & \vdots & \vdots & \ddots & \vdots \\ 0 & 0 & 0 & \cdots & 1 \\ 0 & 0 & 0 & \cdots & 0\end{array}\right], \boldsymbol{B}_{1}=\left[\begin{array}{c}0 \\ \vdots \\ \kappa \\ 0\end{array}\right], \Omega_{1}=\left[\begin{array}{c}0 \\ \vdots \\ 0 \\ \dot{\varpi}\end{array}\right]$, $\boldsymbol{C}_{1}=[1,0, \cdots, 0,0]_{1 \times(m+1)}$.

Based on (3), the observer is designed as

$$
\dot{\hat{\boldsymbol{\nu}}}=\boldsymbol{A}_{1} \hat{\boldsymbol{\nu}}+\boldsymbol{B}_{1} u+\boldsymbol{L}\left(\boldsymbol{C}_{1} \boldsymbol{\nu}-\boldsymbol{C}_{1} \hat{\boldsymbol{\nu}}\right),
$$

where $\boldsymbol{L}=\left[l_{1}, l_{2}, \cdots, l_{m+1}\right]^{T}$ are observer gains. The sliding manifold is designed as $s=\sum_{i=1}^{m} \beta_{i} \hat{\nu}_{i}$, where $\beta_{i}, i=$ $1, \cdots, m-1$ are selected such that the polynomial $\lambda^{m-1}+$ $\beta_{m-1} \lambda^{m-2}+\cdots+\beta_{2} \lambda+\beta_{1}$ is Hurwitz, $\beta_{m}=1$. The outputfeedback controller is given by

$$
u=-\frac{1}{\kappa}\left(\sum_{i=1}^{m} \beta_{i} \hat{\nu}_{i+1}+k_{1} \operatorname{sgn}(s)+k_{2} s\right),
$$

where $k_{1}=\rho+\left|\nu_{1}-\hat{\nu}_{1}\right| \sum_{i=1}^{m} l_{i} \beta_{i}, \rho>0, k_{2}>0$.

\section{Motivations}

The traditional output-feedback SMC can remove the offset caused by mismatched disturbances effectively. However, through the coordinate transformation given by (2) and (3), all unknown states are lumped into $\varpi$ including system states and disturbances. Large observer poles are required to obtain satisfactory performance, which needs large gains. It is natural that large gains will amplify measurement noises, and there will be high frequency fluctuations in estimations and control signal. The high frequency switching of controller may excite the unmodelled dynamics of the system and cause adverse effects on actuator and even destabilize the system.

\section{MAIn RESUlts}

\section{A. Controller Design}

Motivated by [19], the steady states of system (1) can be determined by the following regulator equations:

$$
\begin{aligned}
\dot{\pi}_{i}(t) & =\sum_{j=1}^{n} a_{i j} \pi_{j}(t)+\omega_{i}(t), i=1,2, \cdots, n-1, \\
\dot{\pi}_{n}(t) & =\sum_{j=1}^{n} a_{n j} \pi_{j}(t)+\pi_{u}(t)+\omega_{n}(t), \\
0 & =\pi_{1}(t)-r(t),
\end{aligned}
$$

where $\pi_{i}(t)(i=1,2, \cdots, n)$ and $\pi_{u}(t)$ represent the steady states of system states $x_{i}(i=1,2, \cdots, n)$ and the controller. Obviously, the regulator equations given by (6) are impossible to solve with unknown $\omega_{i}(t)$. To overcome this problem, this section will utilize observation technique and sliding mode theory to design a controller, which will only need the computable $\pi_{1}(t)=r(t)$.

According to system model (1) and (6), the invariant manifold is introduced as

$$
\overline{\mathcal{E}}=\boldsymbol{x}-\Pi
$$

where $\overline{\mathcal{E}}=\left[\varepsilon_{1}, \varepsilon_{2}, \cdots, \varepsilon_{n}\right]^{T}, \varepsilon_{i}=x_{i}(t)-\pi_{i}(t), i=$ $1,2, \cdots, n, \Pi=\left[\pi_{1}(t), \pi_{2}(t), \cdots, \pi_{n}(t)\right]^{T}$. Based on the invariant manifold (7), system (1) can be transformed into

$$
\dot{\overline{\mathcal{E}}}=\boldsymbol{A} \overline{\mathcal{E}}+\boldsymbol{B}\left(u-\pi_{u}(t)\right)
$$

It can be observed from (8) that the mismatched disturbances in (1) are lumped into $\pi_{u}(t)$, which is matched disturbance and needs to be compensated in the controller.

Assumption 1. For system (8), the lumped disturbance $\pi_{u}$ and its derivative are supposed to be bounded and satisfy $\lim _{t \rightarrow \infty} \dot{\pi}_{u}=0$.

Denote $\varepsilon_{n+1}(t)=\pi_{u}(t)$ as an extended state. Let $\mathcal{E}=$ $\left[\overline{\mathcal{E}}^{T}, \varepsilon_{n+1}(t)\right]^{T}$. The augmented system is

$$
\begin{aligned}
\dot{\mathcal{E}} & =\overline{\boldsymbol{A}} \mathcal{E}+\overline{\boldsymbol{B}}_{u} u+\boldsymbol{N} \varsigma, \\
e & =\overline{\boldsymbol{C}} \mathcal{E},
\end{aligned}
$$

where $\overline{\boldsymbol{A}}=\left[\begin{array}{cc}\boldsymbol{A} & -\boldsymbol{B} \\ 0 & 0\end{array}\right], \overline{\boldsymbol{B}}_{u}=\left[\begin{array}{c}\boldsymbol{B} \\ 0\end{array}\right], \overline{\boldsymbol{C}}=[\boldsymbol{C}, 0], \boldsymbol{N}=$ $\left[\mathbf{0}_{1 \times n}, 1\right]^{T}, \varsigma=\dot{\pi}_{u}(t)$. 
Based on (9), an extended state observer is designed as

$$
\begin{aligned}
& \dot{\hat{\mathcal{E}}}=\overline{\boldsymbol{A}} \hat{\mathcal{E}}+\overline{\boldsymbol{B}}_{u} u+\boldsymbol{L}\left(x_{1}-\pi_{1}-\hat{e}\right), \\
& \hat{e}=\overline{\boldsymbol{C}} \hat{\mathcal{E}},
\end{aligned}
$$

where $\hat{\mathcal{E}}=\left[\hat{\varepsilon}_{1}, \hat{\varepsilon}_{2}, \cdots, \hat{\varepsilon}_{n+1}\right]^{T}, \hat{\varepsilon}_{1}, \hat{\varepsilon}_{2}, \cdots, \hat{\varepsilon}_{n+1}$ are estimations of $\varepsilon_{1}, \varepsilon_{2}, \cdots, \varepsilon_{n+1}$ in (9), and $\boldsymbol{L}=\left[l_{1}, l_{2}, \cdots, l_{n+1}\right]^{T}$ are observer gains to be determined. The sliding manifold is designed as

$$
s=\sum_{i=1}^{n} \beta_{i} \hat{\varepsilon}_{i},
$$

with $\beta_{n}=1$ and $\beta_{i}(i=1,2, \cdots, n-1)$ are parameters to be designed. The sliding mode controller is constracted as

$$
u=-\sum_{i=1}^{n} \sum_{j=1}^{n} \beta_{i} a_{i j} \hat{\varepsilon}_{j}-k_{1} \operatorname{sgn}(s)-k_{2} s,
$$

where $k_{1}=\rho+\left|x_{1}-\pi_{1}-\hat{\varepsilon}_{1}\right| \sum_{i=1}^{n} \beta_{i} l_{i}, k_{2}>0$, and $\rho>0$.

Remark 1. The solutions of the regulator equations (6) are not needed in implementation. From the invariant manifold (7), the augmented system (9), the observer and controller given by (10)-(12), it is known that although we cannot calculate $\pi_{i}(t), i=2,3, \cdots, n$ due to the presence of unknown disturbances, the controller can be implemented based on the invariant manifold and observation technique.

\section{B. Stability Analysis}

Taking the derivative of the sliding manifold along (11) and substituting observer (10) and controller (12), one obtains

$$
\begin{aligned}
\dot{s}= & \beta_{1} \dot{\hat{\varepsilon}}_{1}+\beta_{2} \dot{\hat{\varepsilon}}_{2}+\cdots+\dot{\hat{\varepsilon}}_{n}, \\
= & \sum_{i=1}^{n-1} \beta_{i}\left(\sum_{j=1}^{n} a_{i j} \hat{\varepsilon}_{j}+l_{i}\left(x_{1}-\pi_{1}-\hat{e}\right)\right) \\
& +\sum_{j=1}^{n} a_{n j} \hat{\varepsilon}_{j}+l_{n}\left(x_{1}-\pi_{1}-\hat{e}\right)+u \\
= & -k_{1} \operatorname{sgn}(s)-k_{2} s+\sum_{i=1}^{n} \beta_{i} l_{i}\left(x_{1}-\pi_{1}-\hat{e}\right) .
\end{aligned}
$$

Define a candidate Lyapunov function $V=\frac{1}{2} s^{2}$. Taking derivative of $V$ and substituting (13), we have

$$
\begin{aligned}
\dot{V} & =s \dot{s} \leq-k_{1}|s|-k_{2} s^{2}+|s| \sum_{i=1}^{n} \beta_{i} l_{i}\left(x_{1}-\pi_{1}-\hat{e}\right) \\
& <-\rho|s|-k_{2} s^{2}=-\sqrt{2} \rho V^{1 / 2}-2 k_{2} V .
\end{aligned}
$$

Therefore, system states will reach the sliding manifold in a finite time $T_{r}$ with $T_{r} \leq \frac{1}{k_{2}} \ln \left(1+\frac{\sqrt{2} k_{2}}{\rho} V^{1 / 2}(s(0))\right)$ [1].

Denote $\tilde{\boldsymbol{e}}=\mathcal{E}-\hat{\mathcal{E}}=\left[\tilde{e}_{1}, \tilde{e}_{2}, \cdots, \tilde{e}_{n}\right]^{T}$. Once the sliding manifold is reached, one has $s=\sum_{i=1}^{n} \beta_{i} \hat{\varepsilon}_{i}=0$, then

$$
\varepsilon_{n}=\tilde{e}_{n}+\hat{\varepsilon}_{n}=\tilde{e}_{n}-\sum_{i=1}^{n-1} \beta_{i} \hat{\varepsilon}_{i}=\tilde{e}_{n}-\sum_{i=1}^{n-1} \beta_{i}\left(\varepsilon_{i}-\tilde{e}_{i}\right) .
$$

Denote $\overline{\mathcal{E}}_{n-1}=\left[\varepsilon_{1}, \varepsilon_{2}, \cdots, \varepsilon_{n-1}\right]^{T}, \zeta=\left[\overline{\mathcal{E}}_{n-1}^{T}, \tilde{\boldsymbol{e}}^{T}\right]^{T}$. The closed-loop system is written in the following compact form

$$
\dot{\zeta}=\boldsymbol{\Theta} \zeta+\mathbf{\Psi} \varsigma,
$$

where $\boldsymbol{\Theta}=\left[\begin{array}{cc}\tilde{\boldsymbol{A}} & \boldsymbol{a}_{\boldsymbol{n}} \boldsymbol{\beta} \\ 0 & \overline{\boldsymbol{A}}-\boldsymbol{L} \overline{\boldsymbol{C}}\end{array}\right], \boldsymbol{\Psi}=\left[\begin{array}{c}\mathbf{0}_{(2 n-2) \times 1} \\ 1\end{array}\right], \tilde{\boldsymbol{A}}=$ $\overline{\boldsymbol{A}}_{n-1}-\boldsymbol{a}_{\boldsymbol{n}} \boldsymbol{\beta}_{n-1}, \boldsymbol{a}_{\boldsymbol{n}}=\left[a_{1 n}, a_{2 n}, \cdots, a_{(n-1) n}\right]^{T}, \boldsymbol{\beta}=$ $\left[\beta_{1}, \beta_{2}, \cdots, \beta_{n-1}\right], \quad \overline{\boldsymbol{A}}_{n-1}=\left[\boldsymbol{a}_{\mathbf{1}}, \cdots, \boldsymbol{a}_{\boldsymbol{n}-1}\right], \quad \boldsymbol{a}_{i}=$ $\left[a_{1 i}, \cdots, a_{(n-1) i}\right]^{T}$ for $i=1,2, \cdots, n-1$.

Based on the analysis above, we have the following theorem.

Theorem 1. For bounded $\varsigma$, if the observer gains in (10) and the controller parameters in (11) are chosen such that $\bar{A}-$ $\boldsymbol{L} \overline{\boldsymbol{C}}$ and $\tilde{\boldsymbol{A}}$ are Hurwitz matrices, then the output tracking error of system (1) and the estimation errors will converge to a bounded neighbourhood of the origin and the ultimate bound can be made arbitrarily small. If $\varsigma$ tends to zero as time goes to infinity, then the closed-loop system (16) is globally exponentially stable.

Proof. By the input-to-state stability (ISS) [22], there exist a class of $\mathcal{K} \mathcal{L}$ function $\alpha_{1}$ and a class of $\mathcal{K}$ function $\alpha_{2}$ such that for any initial state $\zeta(0)$ and bounded $\varsigma(t)$, the solution of system (16) satisfies $\|\zeta(t)\| \leq \alpha_{1}(\|\zeta(0)\|, t)+$ $\alpha_{2}\left(\sup _{0<\tau<t}|\varsigma(\tau)|\right)$ and $\|\zeta(\infty)\| \leq \alpha_{2}(\delta) \leq \infty$ with $|\varsigma(t)| \leq$ $\delta, \delta>0$. Therefore, the output tracking error and the estimation errors are bounded.

Along (9), (10) and (16), the observer estimation error subsystem is depicted by

$$
\dot{\tilde{e}}=(\bar{A}-L \bar{C}) \tilde{e}+N \varsigma .
$$

According to the comparison lemma [22], we have

$$
\tilde{\boldsymbol{e}}(t)=e^{(\overline{\boldsymbol{A}}-\boldsymbol{L} \overline{\boldsymbol{C}}) t} \tilde{\boldsymbol{e}}(0)+\int_{0}^{t} e^{(\overline{\boldsymbol{A}}-\boldsymbol{L} \overline{\boldsymbol{C}})(t-s)} \boldsymbol{N} \varsigma(s) d s .
$$

Without loss of generality, the observer poles are set as $\omega_{0}<0$. Combining Lemma 1, one obtains

$$
\begin{aligned}
\|\tilde{\boldsymbol{e}}(t)\| & \leq \int_{0}^{t}\left\|e^{(\overline{\boldsymbol{A}}-L \overline{\boldsymbol{C}})(t-s)}\right\|\|\boldsymbol{N}\||s(s)| d s+\left\|e^{(\overline{\boldsymbol{A}}-L \overline{\boldsymbol{C}}) t}\right\|\|\tilde{\boldsymbol{e}}(0)\| \\
& \leq \delta \int_{0}^{t} c_{2} e^{\frac{\omega_{o}}{2}(t-s)} d s+c_{1} e^{\frac{\omega_{o}}{2} t}\|\tilde{\boldsymbol{e}}(0)\| \\
& \leq \frac{2 \delta c_{2}\left(1-e^{\frac{\omega_{o}}{2} t}\right)}{-\omega_{o}}+c_{1} e^{\frac{\omega_{o}}{2} t}\|\tilde{\boldsymbol{e}}(0)\|,
\end{aligned}
$$

where $c_{1}$ and $c_{2}$ are positive constants. When time goes to infinity, there is $\lim _{t \rightarrow \infty}\|\tilde{\boldsymbol{e}}(t)\|=\frac{2 \delta c_{2}}{-\omega_{o}}$. If the observer gains are chosen such that the matrix $\bar{A}-\boldsymbol{L} \bar{C}$ is Hurwitz, then the estimation errors are bounded and can be arbitrarily small by amplifying the observer poles.

From (16), the state subsystem is

$$
\dot{\overline{\mathcal{E}}}_{n-1}=\tilde{\boldsymbol{A}} \overline{\mathcal{E}}_{n-1}+\boldsymbol{a}_{\boldsymbol{n}} \boldsymbol{\beta} \tilde{\boldsymbol{e}} .
$$

Based on the comparison lemma [22], we have

$$
\overline{\mathcal{E}}_{n-1}(t)=e^{\tilde{\boldsymbol{A}} t} \overline{\mathcal{E}}_{n-1}(0)+\int_{0}^{t} e^{\tilde{\boldsymbol{A}}(t-s)} \boldsymbol{a}_{\boldsymbol{n}} \boldsymbol{\beta} \tilde{\boldsymbol{e}}(s) d s .
$$

Denote a positive constant $\hbar=\left\|\boldsymbol{a}_{\boldsymbol{n}}\right\|\|\boldsymbol{\beta}\|\|\tilde{\boldsymbol{e}}\|$. The poles of $\tilde{\boldsymbol{A}}$ are set as $\omega_{c}<0$. Similar with (19), it gets

$$
\left\|\overline{\mathcal{E}}_{n-1}(t)\right\| \leq m_{1} e^{\frac{\omega_{c}}{2} t}\left\|\overline{\mathcal{E}}_{n-1}(0)\right\|+\frac{2 m_{2} \hbar}{-\omega_{c}}\left(1-e^{\frac{\omega_{c}}{2} t}\right),
$$

with $m_{1}, m_{2}>0$. Therefore, we have $\lim _{t \rightarrow \infty}\left\|\overline{\mathcal{E}}_{n-1}\right\|=\frac{2 m_{2} \hbar}{-\omega_{c}}$. Noting that the ultimate bound for $\|\tilde{e}\|$ can be made arbitrarily 


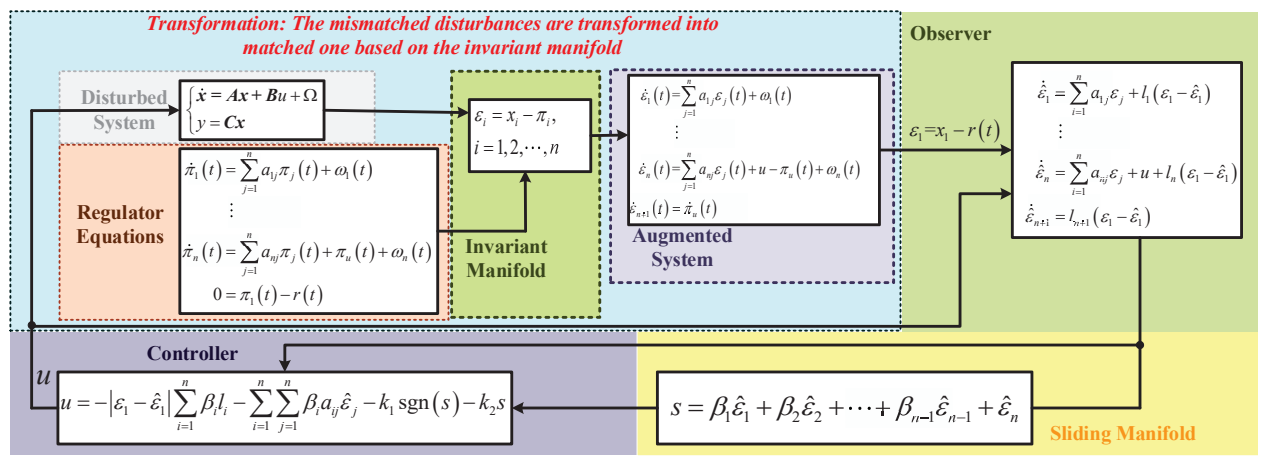

Fig. 1. Block diagram of the proposed invariant manifold based output-feedback SMC.

small by choosing sufficiently large $\omega_{o}$, the ultimate bound for the tracking error can be made arbitrarily small.

Furthermore, if $\varsigma(t)$ tends to zero as $t$ tends to infinity, according to [23], we have

$$
\begin{aligned}
\lim _{t \rightarrow \infty} \tilde{\boldsymbol{e}}(t) & =\lim _{s \rightarrow 0} \tilde{\boldsymbol{E}}(s)=\lim _{s \rightarrow 0}[s \boldsymbol{I}-(\overline{\boldsymbol{A}}-\boldsymbol{L} \overline{\boldsymbol{C}})]^{-1} \boldsymbol{N} \mathcal{L}(\varsigma(t)) \\
& =\lim _{s \rightarrow 0}[s \boldsymbol{I}-(\overline{\boldsymbol{A}}-\boldsymbol{L} \overline{\boldsymbol{C}})]^{-1} \boldsymbol{N} \cdot \lim _{t \rightarrow \infty} \varsigma(t)=\mathbf{0} .
\end{aligned}
$$

Then, based on the above result, one has

$$
\begin{aligned}
\lim _{t \rightarrow \infty} \overline{\mathcal{E}}_{n-1}(t) & =\lim _{s \rightarrow 0}(s \boldsymbol{I}-\tilde{\boldsymbol{A}})^{-1} \boldsymbol{a}_{\boldsymbol{n}} \mathcal{L}\left(\sum_{i=1}^{n} \beta_{i} \tilde{e}_{i}(t)\right) \\
& =\lim _{s \rightarrow 0}(s \boldsymbol{I}-\tilde{\boldsymbol{A}})^{-1} \boldsymbol{a}_{\boldsymbol{n}} \lim _{t \rightarrow \infty} \sum_{i=1}^{n} \beta_{i} \tilde{e}_{i}(t)=\mathbf{0} .
\end{aligned}
$$

Taking (15) in mind, one obtains

$$
\lim _{t \rightarrow \infty} \varepsilon_{n}(t)=\lim _{t \rightarrow \infty} \tilde{e}_{n}-\lim _{t \rightarrow \infty} \sum_{i=1}^{n-1} \beta_{i} \varepsilon_{i}+\lim _{t \rightarrow \infty} \sum_{i=1}^{n-1} \beta_{i} \tilde{e}_{i}=0 .
$$

Since $\varepsilon_{1}=x_{1}-r(t)$, we have $\lim _{t \rightarrow \infty} y(t)=r(t)$. Therefore, the system output tracks the desired signal asymptotically.

The control structure of the proposed method in implementation is shown in Fig. 1.

TABLE I

PARAMETERS OF BUCK CONVERTER-DRIVEN DC MOTOR

\begin{tabular}{c|c|c}
\hline \hline Meanings & Parameters & Nominal value \\
\hline Input voltage & $E$ & $40 \mathrm{~V}$ \\
\hline Converter inductor & $L$ & $10 \mathrm{mH}$ \\
\hline Converter capacity & $C$ & $1000 \mu \mathrm{F}$ \\
\hline Load resistor & $R$ & $250 \Omega$ \\
\hline Armature inductor & $L_{a}$ & $2 \mathrm{mH}$ \\
\hline Armature winding resistor & $R_{a}$ & $1.45 \Omega$ \\
\hline Viscous friction coefficient & $b$ & $65.12 \times 10^{-6}$ \\
\hline Moment of inertia of rotor & $J$ & $32.5 \times 10^{-6}$ \\
\hline Torque constant & $k_{m}$ & 0.0699 \\
\hline Counter electromotive force constant & $k_{e}$ & 0.0699 \\
\hline \hline
\end{tabular}

\section{ApPliCATIONS}

A converter-driven DC motor system shown in Fig. 2 is employed in experiments, and the mathematical model is

$$
\begin{aligned}
& \frac{d i_{L}}{d t}=-\frac{v_{o}}{L}+\frac{E}{L} \mu, \frac{d v_{o}}{d t}=\frac{i_{L}}{C}-\frac{v_{o}}{R C}-\frac{i_{a}}{C}, \\
& \frac{d i_{a}}{d t}=\frac{v_{o}}{L_{a}}-\frac{R_{a}}{L_{a}} i_{a}-\frac{k_{e}}{L_{a}} \omega, \frac{d \omega}{d t}=\frac{k_{m}}{J} i_{a}-\frac{b}{J} \omega-\frac{\tau_{L}}{J},
\end{aligned}
$$

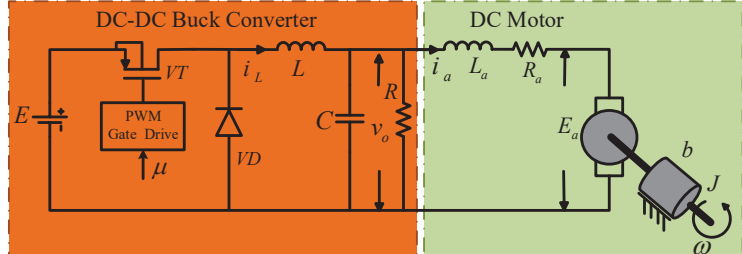

Fig. 2. Typical circuit of converter driven DC motor system.

where $i_{L}$ is the converter inductor current, $v_{o}$ the converter output voltage, the duty ratio $\mu \in[0,1]$ is the control input, $i_{a}$ is the armature circuit current, $\omega$ the angular velocity of DC motor. $\tau_{L}$ is the unknown load torque, which is the mismatched disturbance. The physical meanings and nominal values of the parameters are listed in Table I.

In experiments, the traditional output-feedback SMC is employed for comparisons, which is designed following the process given by (2)-(5). The proposed controller is

$$
\begin{aligned}
\mu= & -\frac{L}{E}\left[-\left(\frac{1}{L} \hat{\varepsilon}_{1}+\frac{E}{L} \hat{\varepsilon}_{5}\right)+\beta_{2}\left(\frac{1}{C} \hat{\varepsilon}_{1}-\frac{1}{R C} \hat{\varepsilon}_{2}-\frac{1}{C} \hat{\varepsilon}_{3}\right)\right. \\
& +\beta_{3}\left(\frac{1}{L_{a}} \hat{\varepsilon}_{2}-\frac{R_{a}}{L_{a}} \hat{\varepsilon}_{3}-\frac{k_{e}}{L_{a}} \hat{\varepsilon}_{4}\right)+\beta_{4}\left(\frac{k_{m}}{J} \hat{\varepsilon}_{3}-\frac{b}{J} \hat{\varepsilon}_{4}\right) \\
& \left.+k_{1} \operatorname{sgn}(s)+k_{2} s\right],
\end{aligned}
$$

with the sliding manifold $s=\hat{\varepsilon}_{1}+\beta_{2} \hat{\varepsilon}_{2}+\beta_{3} \hat{\varepsilon}_{3}+\beta_{4} \hat{\varepsilon}_{4}$, and $k_{1}=\rho+\left|\omega-\omega^{*}-\hat{\varepsilon}_{4}\right| \sum_{i=1}^{4} \beta_{i} l_{i}, \rho>0, k_{2}>0$. The estimations are obtained from the observer based on (10). The observer poles of the two methods are set as $\omega_{o}=-500$ and the controller poles in the proposed method are $\omega_{c}=-150$ and $\omega_{c}=-300$ in the traditional method. 
(a)

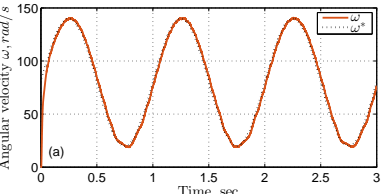

(b)
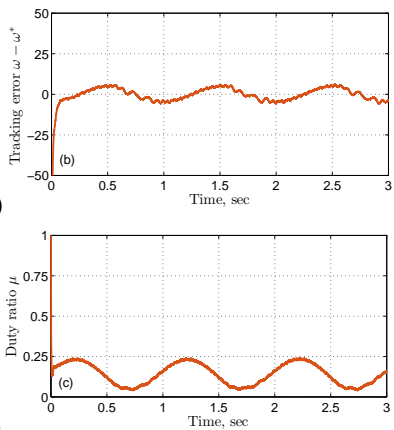

(d)

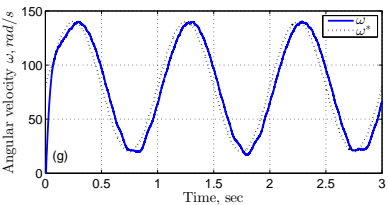

(e)

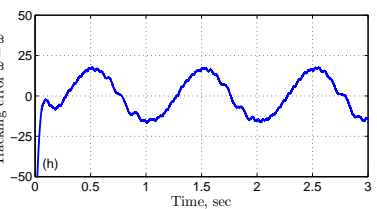

(f)

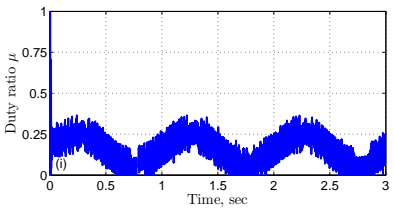

Fig. 3. Response curves under the proposed output feedback SMC method (left) and traditional output feedback SMC (right) for sinusoidal signal tracking (top: angular velocity; middle: tracking error; bottom: duty ratio).

(a)
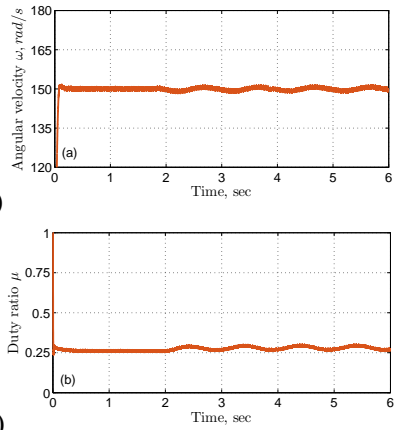

(b)

(c)

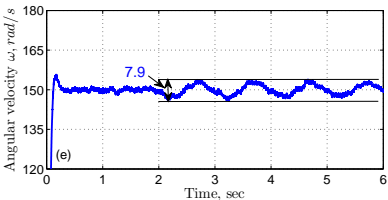

d)

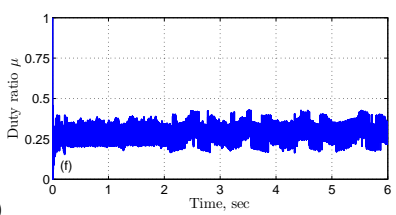

Fig. 4. Response curves under the proposed output feedback SMC method (left) and traditional output feedback SMC (right) in the presence of timevarying load torque disturbance (top: angular velocity; bottom: duty ratio)

Fig. 3 shows the experimental results in tracking sinusoidal reference signal $\omega^{*}=80+75 \sin (2 \pi t) \mathrm{rad} / \mathrm{s}$. It can be observed that larger poles are required for traditional method to obtain similar tracking performance with the proposed controller. As shown in Fig. 3(c) and Fig. 3(f), the frequently switching in control signal provided by the traditional method is more serious than the controller proposed in this paper. In experimental results shown by Fig. 4, the unknown time-varying load torques are imposed on motor shaft. The reference signal is $150 \mathrm{rad} / \mathrm{s}$, and the unknown load torque changes from 0 to $\tau_{L}=0.05 \sin (2 \pi t)+0.05(\mathrm{~N} \cdot \mathrm{m})$ at $2 \mathrm{~s}$. The proposed controller provides much smaller speed fluctuations than the traditional method, which validates the better disturbance rejection ability of the proposed method.

\section{CONCLUSION}

An invariant manifold based output-feedback SMC method has been proposed in this paper for systems with mismatched disturbances. An observer has been designed to reconstruct unmeasurable states by means of the augmented system derived from invariant manifold. Under the controller, system output tracks the reference signal asymptotically even in the presence of mismatched disturbances. Experimental results have validated the effectiveness of the proposed controller.

\section{REFERENCES}

[1] S. Yu, X. Yu, B. Shirinzadeh, and Z. Man,"Continuous finite-time control for robotic manipulators with terminal sliding mode", Automatica, vol. 41, no. 11, pp. 1957-1964, 2005.

[2] Z. Chen, Y. Chen, and Z. Yan, "Simplified hysteresis sliding-mode control for superbuck converter," IEEE Trans. Circuits Syst. II, Exp. Briefs, DOI: 10.1109/TCSII.2020.2979796.

[3] Y. Yildiz, A. Sabanovic, and K. Abidi, "Sliding-mode neuro-controller for uncertain systems," IEEE Trans. Ind. Electron., vol. 54, no. 3, pp. 1676-1685, 2007.

[4] P. Roy, B.K. Roy, "Sliding mode control versus fractional-order sliding mode control: applied to a metnetic levitation system," J. Control Autom. Electr. Syst., vol. 31, no. 3, pp. 597-606, 2020.

[5] K. Zhang, G.-R. Duan, M.-D. Ma, "Dynamic output feedback sliding mode control for spacecraft hovering without velocity measurements," $J$. Frankl. Inst., vol. 356, pp. 1991-2014, 2019.

[6] S. H. Chincholkar, W. Jiang , and C.-Y. Chan, "A normalized output errorbased sliding-mode controller for the DC-DC cascade boost converter," IEEE Trans. Circuits Syst. II, Exp. Briefs, vol. 67, no. 1, pp. 92-96, 2020.

[7] Y. Gao, B. Sun, and G. Lu, "Passivity-based integral sliding-mode control of uncertain singularly perturbed systems," IEEE Trans. Circuits Syst. II, Exp. Briefs, vol. 58, no. 6, pp. 386-390, 2011.

[8] J. Yang, S. Li, and X. Yu, "Sliding-mode control for systems with mismatched uncertainties via a disturbance observer," IEEE Trans. Ind. Electron., vol. 60, no. 1, pp. 160-169, 2013.

[9] Y. Zhao, S. Yu, and J. Lian, "Anti-disturbance bumpless transfer control for switched systems with its application to switched circuit model," IEEE Trans. Circuits Syst. II, Exp. Briefs, DOI: 10.1109/TCSII.2020.2970068.

[10] X.-H. Chang , R.-R. Liu, and J. H. Park, "A further study on output feedback $H_{\infty}$ control for discrete-time systems," IEEE Trans. Circuits Syst. II, Exp. Briefs, vol. 67, no. 2, pp. 305-309, 2020.

[11] Y. Zhao, J. Zhao, J. Fu, Y. Shi, and C. Chen, "Rate bumpless transfer control for switched linear systems with stability and its application to aero-engine control design," IEEE Trans. Ind. Electron., vol. 67, no. 6, 2020.

[12] Z. Zhao, J. Yang, S. Li, X. Yu, and Z. Wang, "Continuous output feedback TSM control for uncertain systems with a DC-AC inverter example," IEEE Trans. Circuits Syst. II, Exp. Briefs, vol. 65, no. 1, 2018.

[13] A. Levant, "Higher-order sliding modes, differentiation and outputfeedback control," Int. J. Control, vol. 76, no. 9-10, 2003.

[14] J. Han, "From PID to active disturbance rejection control," IEEE Trans. Ind. Electron., vol. 56, no. 3, pp. 900-906, 2009.

[15] Y. Xia, P. Fan, M. Fu, and L. Ye, "Modeling and compound control for unmanned turret system with coupling," IEEE Trans. Ind. Electron., vol. 63, no. 9, pp. 5794-5803, 2016.

[16] J. Mao, J. Yang, S. Li, Y. Yan, and Q. Li, "Output feedback-based sliding mode control for disturbed motion control systems via a higherorder ESO approach," IET Control Theory Appl., vol. 12, no. 15, pp. 2118-2126, 2018

[17] J. A. Moreno, "Discontinuous integral control for mechanical systems," in Proc. IEEE 14th Int. Workshop Variable Struct. Syst., Nanjing, China, Jun. 01-04, 2016, pp. 142-147.

[18] L. Oliveira, A. Bento, V.J.S. Leite, F. Gomide, "Evolving granular feedback linearization: Design, analysis, and applications," Appl. Soft Comput., vol. 86, pp. 105927, 2020.

[19] A. Isidori and C. Byrnes, "Output regulation of nonlinear systems," IEEE Trans. Autom. Control, vol. 35, no. 2, pp. 131-140, 1990.

[20] W. Zhang, Stability of networked control systems, PhD. thesis, Case Western Reserve University, Department of Electrical Engineering and Computer Science, 2001.

[21] J. P. V. S. Cunha, R. R. Costa, F. Lizarralde, and L. Hsu, "Peaking free variable structure control of uncertain linear systems based on a high-gain observer," Automatica, vol. 45, pp. 1156-1164, 2009.

[22] H. Khalil, Nonlinear Systems, third edition. Upper Saddle River, NJ, USA: Prentice-Hall, 2002.

[23] S. Li, J. Yang, W. H. Chen, and X. Chen, "Generalized extended state observer based control for systems with mismatched uncertainties," IEEE Trans. Ind. Electron., vol. 59, no. 12, pp. 4792-4802, 2012. 